\title{
Human factors at sea: common patterns of error in groundings and collisions
}

\author{
Dr Carl Macrae \\ ESRC Centre for Analysis of Risk and Regulation, London School of Economics and \\ Political Science
}

\begin{abstract}
This research aimed to identify and map the common patterns of human and organizational causes underlying two types of marine accident: groundings and collisions. Generalising patterns of causality from relatively unique and individual accident events required a structured and exploratory analytical approach. Two complementary human factor analysis tools were employed to analyse a set of 30 detailed marine accident reports produced by the Australian Transport Safety Bureau. Common patterns of causality were identified for both groundings and collisions. Groundings commonly resulted from a failure to adequately plan a passage, coupled with either a problem locating the vessel, or communication problems on the bridge. Collisions often involved a fishing vessel and a bulk carrier or cargo vessel, and commonly resulted from both a problem identifying the existence or speed of the other vessel and, again, an inadequate planning process. Generalising these common causal patterns from a number of accidents identifies a range of points at which crews, managers and policymakers can intervene to forestall the development of these accidents. The method developed here may also be productively extended and applied to other accident types and used as an ongoing risk management tool.
\end{abstract}




\section{Human factors at sea: common patterns of error in groundings and collisions}

\section{Introduction}

Understanding the human and organizational factors underlying major shipping accidents is a topic of key importance for maritime policy and management. Accidents at sea can cause severe harm, leading to loss of life, environmental damage and serious financial costs. As such, they are typically investigated extensively in order to learn from them and to improve risk management, both within individual companies and across the industry. Accident investigations often focus on the role of human error---the mistakes, unsafe acts or violations committed by crew members. Studies consistently estimate that around $80 \%$ of causes in marine accidents are attributable to human factors $[1,2]$. Human error has traditionally been viewed as an individual cognitive, behavioural or sometimes moral issue, caused merely by carelessness or ignorance. Increasingly, however, there is a growing recognition of the influence of organizational context and situational factors in provoking and shaping errors. Situational factors such as inappropriate equipment or clumsy procedures can provide routine error traps for people to fall into. Likewise, organizational factors, such as production pressures or decisions that leave crew under-resourced, equally set the stage for poor performance [3]. As a leading scholar in this field, James Reason [4], has pithily observed, to understand and manage human error we need to focus less on the human condition and more on the conditions in which people work.

Understanding the complex human, situational and organizational factors underlying past accidents is centrally important for improving safety and risk management in the industry. However, learning from past accidents is challenging. The sequences of events leading to an accident often appear unique and individual to each specific case. We therefore run the risk of drawing lessons that are equally specific and fail to generalise to other circumstances and situations---akin to standing around examining the stable door long after the horse has bolted. To learn from past accidents, we need to generalise from them, drawing systematic, general and widely applicable insights into their causes, and mapping causal patterns across several levels of analysis [5]. This article attempts this by examining two types of shipping accident---groundings and collisions---to identify the common patterns of human 
factor events that can cause them. To do this, 30 detailed marine accident investigation reports produced by the Australian Transport Safety Bureau are analysed using two complementary human factor analysis methods. Through this analysis, generalised accident scenarios are developed for both collisions and groundings, isolating the key causal patterns involved in each accident type. This article argues that producing these generalised accident scenarios provides important practical benefits. They can help crews recognise and control hazardous shipboard situations as they emerge. They can support company managers in analysing and addressing gaps in their internal risk management systems. And they can aid policy makers in identifying the most productive areas to target with regulatory interventions.

The remainder of this article is structured as follows. First, some of the key ideas relating to the analysis of marine accidents and human factors are examined. Second, the methods developed to generalise marine accidents are detailed. Next, the findings of this research are presented and discussed, examining the common forms of error in groundings and collisions and how these are typically caused. Conclusions are then drawn concerning the implications and limitations of this approach to learning from accidents, the possibility of its wider application within the industry and directions for future research.

\section{Analysing errors and accidents}

Finding the appropriate level of analysis at which to understand major accidents has long been a key issue in discussions of risk and safety management [6]. These challenges apply equally to accidents in the maritime industry as much as any other. At one extreme, accidents appear to be idiosyncratic and one-off events, each concerning a unique set of problems that came together on the day in an entirely unpredictable and surprising way. At the other extreme, models of accidents can be so abstract as to be relatively imprecise and impractical, focusing on a small number of intangible concepts such as 'complexity' or 'coupling' within social-technical work systems [7]. Whilst analysis at each of these levels has its uses, a balance between the two has to be found to allow general lessons to be drawn that can guide practical management and policy responses. To this end, the most common and productive approaches to analysing accidents focus on mapping chains of causality leading up to the event. To analyse the role of human factors in accidents there are two distinct but complementary approaches in use---organizational error analysis and human 
reliability analysis. Both have been applied with useful results in the marine industry, and each underlies one of the two analytical tools used in the research presented here.

\section{Organizational error analysis}

Organizational error analysis has its roots in cognitive and 'error' psychology, and aims to analyse both the form and causes of unsafe acts and their relationship to safety defences and risk controls. Early analyses focused largely on identifying the form of common errors in marine accidents [8]. For instance, one human error analysis [9] found that $76 \%$ of human errors implicated in shipping accidents occur on the bridge. Stoop [10] examined a range of types of marine accidents, including groundings and collisions, in order to find common human and organizational factors involved. He found that the principle causes for both often involved inaccurate position finding, little or no use of navigation aids, poor communication on the bridge and inadequate preparation for the journey, including the absence of adequate or up to date charts.

The scope of this approach to human factors was significantly broadened by Reason's work on organizational error. Reason's $[4,6]$ model explains how safety defences and barriers are breached by human factors. Defences may either take the form of 'hard', physical barriers guarding against hazards, or they may be 'soft', such as regulations or standard operating procedures. A basic assumption is that no defence is perfect, and when an error or unsafe act coincides with a defensive weakness, an accident may result. In this model, accidents are caused by both 'active failures' and 'latent conditions'. Active failures are the unsafe acts committed by those working at the sharp-end---the crew. These unsafe acts are caused by both cognitive and situational factors, the latter of which are in turn directly shaped by broader organizational conditions. For instance, management decisions may result in the provision of inappropriate equipment, leading to the invention of a clumsy work-around solution making mistakes more likely. Latent conditions act directly upon safety defences. Shortcomings or poor decisions in the upper levels of organizations---such as implementing a deficient training regime---may leave gaps in defences.

This approach to analysing human factors also makes an important distinction between errors and violations. Errors relate to some form of cognitive lapse resulting from, for instance, inattention, misperception or inadequate knowledge. Violations, on the other hand, involve actively deviating from prescribed procedures. As such, 
they involve a motivational aspect. Violations may be routine---whereby deviations are habitual---or erroneous, when the individual is unaware of the correct procedure. Both errors and violations have been extensively classified $[4,10]$. However, as has been argued in other analyses of shipping accidents, more detailed frameworks are often of little use in practice due to the lack of detailed causal information that is required to apply them [11].

\section{Human reliability analysis}

Human Reliability Analysis (HRA) has its roots in engineering risk analysis, and aims to quantitatively predict likely failure event sequences [12]. To analyse human factors in shipping accidents, error frequency databases and expert opinion have been used to predict reasons both for collision [13] and grounding [14]. Technica's [13] analysis of collisions proposed the most likely scenario as that of the 'errant vessel', with inadequate watch keeping due to the bridge crew being distracted, incapacitated or incompetent. Amrozowicz's [14] grounding analysis predicted powered groundings were the result of either a planning error---due to insufficient or incorrect information---or a detection or action error, whereby deviation from a safe track is either not detected or is incorrectly acted upon. Neither of these analyses went beyond these individual human errors to consider the likely underlying cognitive, situational or organizational factors. Admittedly, however, this was not their aim. Again, this HRA approach has recently been developed further. Hollnagel [15] developed a model that aims to account for the situational influences on human action and, specifically, error. The model uses local conditions and task-specific factors to categorise errors. So in this approach cognitive failures are traced back to their psychological and situational precursors, though relatively limited attention is paid to the broader organizational conditions involved.

Organizational error analysis and HRA provide complementary approaches to analysing human factors and modelling accident causation. To date, both of these approaches are typically used to analyse the causal sequences underlying individual accidents, or to quantitatively summarise the frequency of errors in different types of accidents. This research, however, aims to apply these approaches to a set of shipping accidents in order to identify the typical patterns of organizational failure involved. That is, it aims to answer the question, what do the common causal patterns underlying a grounding and a collision typically look like? 


\section{Methods: generalising marine accidents}

This research aimed to isolate the causal patterns underlying two types of marine accident: groundings and collisions. The aim was to pragmatically model how the typical causal patterns were structured across the levels of organizational conditions, situational and psychological factors, unsafe acts and defensive failures. Two complementary investigative tools were used to this end, each drawing on one of the two human factors analysis approaches discussed previously. Aspects of each of these analytical approaches were integrated to produce a framework within which Generalised Accident Scenarios (GAS) could be built (figure 1).

\section{Insert figure 1 about here.}

This analysis of common causal patterns aimed more for a heuristic, pragmatic model of causation than for any claims of comprehensiveness or precision. That is, the relationships between the different factors analysed did not represent deterministic cause-consequence links, but instead the likely and most frequent associations.

\section{Materials}

A set of 30 Australian Transport Safety Bureau (ATSB) marine accident reports, produced between 1995 and 2000, were used for the analysis. 15 collisions and 15 groundings were selected through stratified random sampling from the reports publicly available on the ATSB website. ATSB reports are well suited for re-analysis, given that they are all produced in a standard format, contain extensively detailed and non-judgemental analysis, and during this five year period were the product of similar investigation procedures.

\section{Analytical tools}

The two analytical tools employed to analyse the accident reports were the Cognitive Reliability and Error Analysis Method (CREAM) [15] and the Marine Accident Investigation Tool (MAIT: formerly RAIT, Railway Accident Investigation Tool) [16]. Each consists of an underlying model, classification scheme and methodology. 


\section{Marine Accident Investigation Tool}

Originally developed for the railway industry, MAIT's principles and classification are derived from Reason's [4, 6] organizational error model, and therefore are highly transferable across domains. Beginning at the adverse outcome, MAIT traces the human factor elements along both latent and active pathways to ten root organizational conditions, called Marine Problem Factors (MPF), figure 2.

Insert figure 2 about here.

MAIT analysis provides a structured way to identify the defensive, individual and situational factors leading to an accident, and then assess the relative importance of each MPF for their occurrence. Analysis proceeds in six stages.

i. The accident outcome is described: fatality, injury, damage or near miss.

ii. Latent defensive weaknesses are identified and categorised according to the defensive mode and function they impact (figure 3).

iii. Unsafe acts are identified and classified as errors, violations or erroneous violations.

iv. The tasks associated with these unsafe acts are identified and detailed.

v. Local contributing factors are isolated and classified as either personal or situational.

vi. Finally, the relative contribution of each of the ten MPFs are evaluated, both in terms of their impact on defensive weaknesses (i.e. the latent pathway, from (ii)) and local factors (i.e. the active pathway, from (v)). Ratings are made on a scale of $o$ (no contribution) to 4 (vary large contribution). The ratings are then summed for each MPF to give an active and latent failure profile.

MAIT's formal output is this MPF profile, highlighting the most important organizational failures for active and latent pathways. Further, in the course of the analysis, extensive qualitative data is generated detailing situational, defensive and error factors involved in each accident. 
Insert figure 3 about here.

Cognitive Reliability and Error Analysis Method

CREAM analyses contextual influences on human action and was developed as a 'second generation' HRA method [15]. It consists of an extensive and nonhierarchical classification scheme and a formalised method specifying how to consequences and antecedents, to explore causal patterns.

First, the model categorises broad 'error modes' (e.g. distance---too far; timing---to late). Causal antecedents of these errors are then classified within fourteen groups. For convenience here, these can be divided into person, technology and organization related causes (figure 4).

Insert figure 4 about here.

Within each group, antecedents are specified at two levels, the general and the specific. For instance, a general Permanent Person Related Function may be 'cognitive bias', which can then be broken down into its specific form (e.g. 'focus gambling', 'incorrect revision of probabilities', 'hindsight bias', 'attribution error', 'illusion of control', 'confirmation bias' and 'hypothesis fixation').

Further, within the model, each antecedent has predetermined links to other general and specific antecedents. So, analysis proceeds by identifying the error mode then linking it to one of the general antecedents, at which point a more detailed categorisation can take place. Links to the next antecedent can then be followed in turn. A causal chain terminates when an antecedent cannot be broken down any further. The output of CREAM analyses takes the form of branching networks of consequent-antecedent chains, leading from each error mode.

\section{Procedure}

Each accident report was analysed with both of the tools in turn, producing a large corpus of data, both quantitative and qualitative. This data was then inductively explored to identify systematic causal patterns within each accident type. The MAIT output was analysed for common defensive, organizational and active failures. Defensive failure frequencies were summed by defensive mode for each accident 
type. The most important failure modes were then explored qualitatively in more detail. Common organizational conditions were examined via MAIT's formal output, the MPF profile. Next, the qualitative data on situational and personal factors were grouped based on similarity within each accident type, and the most frequent factors were identified.

For each accident type, the most frequent proximal (i.e. first level) antecedents isolated by CREAM were identified. The most common proximal antecedents for each type were then explored in more depth. The second level antecedents for each were recorded and an event tree produced, and compared within each type of accident. This identified the immediate human and situational antecedents for each accident type.

Finally, the data produced from the above, supplemented by the qualitative information generated during the MAIT and CREAM analyses, was used to construct a Generalised Accident Scenario for each accident type, by mapping the most common causal patterns and tracing the most likely links between the defensive failures and the organizational, situational and human factors involved.

\section{Results and discussion: common patterns of error}

Significant similarities were found in terms of the underlying causal factors for both groundings and collisions. Common patterns of error could be mapped for both accident types, capturing a large proportion of the observed causal factors involved. Groundings commonly resulted from a failure to adequately plan a passage, coupled with either a problem locating the vessel during the passage, or communication problems on the bridge. That is, if there was a problem with the planning of a passage, then the ship was more likely to succumb to some other disruptive event during the passage. The analysis of collisions conducted here focused on one subtype: collisions between trawlers and a bulk carrier or cargo vessel. These made up the majority of the accident reports that were sampled, and also posed a severe risk of loss of life for trawler crews. Bulk/cargo-trawler collisions commonly resulted from both a problem identifying the existence or speed of the other vessel and, again, an inadequate planning process. However, the planning failures were different on each type of vessel. Trawler crew typically failed in how they planned their watch. Bulk carrier or cargo vessel crew instead tended to plan an inadequate passing clearance. Common patterns of defensive failures, unsafe acts and situational and organizational precursors were associated with the key failure modes just described. 
These patterns, and the outputs from the analytical tools, are detailed in turn next, along with a description of the Generalised Accident Scenario for groundings and collisions.

\section{Groundings}

All the groundings analysed occurred during either approaching or leaving port via channel systems. In such a constrained performance environment, failures and triggering events were themselves constrained. There was little beyond the bridge team's vigilance and awareness, and in particular the performance of the pilot, preventing grounding. Deficits in planning in combination with a communication or ship location problem were sufficient to cause a grounding.

\section{Human factor analysis}

The four major triggering factors isolated by CREAM were planning (30.3\%), interpretation (18.2\%), communication (15.2\%) and team support (12.1\%). These accounted for $75.8 \%$ of all proximal antecedents. The event trees showed that no single one was sufficient for grounding. A combination of planning and either one or two of the others was, however. One of these combinations provided sufficient cause in $53.3 \%$ of all cases of grounding.

\section{Planning errors}

Analysis of the CREAM data revealed that route planning failure divided into wrong (18.1\%), absent (27.3\%) or incomplete (54.6\%) plans. Wrong plans were due almost exclusively to out of date or absent charts. Incomplete and absent plans regularly arose from cognitive bias, (often illusion of control, with an over-reliance on personal experience of the passage) and poor working conditions in the form of time pressures and heavy demand---a factor often highlighted in shipping safety [17]. Constructing a detailed and adequate plan of passage is the requirement of every pilot. As such, $81.9 \%$ of planning failures (incomplete or no plan) resulted from a violation. These were attributed to both cognitive bias and time limitation. Pilots, familiar with their local channels, worked only to superficial plans. They were not deliberately taking risks, but were routinely---and presumably automatically [18]---running them. Pilots also often had little time to complete jobs. Anecdotal accounts in the reports suggest 
that these violations were routine due to the pressured and familiar nature of the task. The relatively infrequent occurrence of bad outcomes can allow these violations to persist---only 41 groundings were investigated by the ATSB from 1991 to 2001, yet pilots at busy ports can make this many passages in a week.

\section{Position finding errors}

Interpretation failures took two forms: decision errors regarding the vessel's heading (33.3\%), and faulty diagnoses of vessel position or channel limits (66.6\%). These were exclusively traced to individual factors of fatigue, insufficient knowledge---for instance, of vessel handling characteristics---or missing or confusing information. Fatigue accounted for $50 \%$ of MAIT situational factors, and misjudging the vessel's location by the pilot made up $57.1 \%$ of the recorded errors.

\section{Communication errors}

Communication failures were entirely due to relevant information not being communicated. Team support failures arose due to a lack of team cohesiveness and an inadequate allocation of tasks---that is, bridge resource management (BRM) [19]. These failures resulted in crew members failing to conduct the appropriate checks and balances on each other's actions. CREAM analysis determined these unsafe acts as due to inadequate knowledge of what it was necessary to communicate, and the lack of adequate procedures to explicitly specify such communications. This is likely to be exacerbated for temporary (i.e. non-crew) pilots, aboard for a short space of time to make the passage. Inadequate task allocation was on the whole due to undermanning on the bridge. MAIT analysis revealed that $41.2 \%$ and $23.5 \%$ of situational factors in groundings were accounted for by poor communication and an undermanned bridge respectively.

\section{Defensive and organizational factors}

MAIT analysis highlighted three important defensive failure modes; protection systems (33.3\%), hazard identification and elimination (19.4\%) and supervision (19.4\%). Breaking these down further, $87.9 \%$ of protection system failures were due to no or poor planning of the passage---supporting the findings above. Just under one-half (48.5\%) of hazard identification failures were due to out of date charts, whilst over a third (37.4\%) were due to inadequate position plotting. $80.4 \%$ of 
supervision failures were due to an absent or inadequate briefing by the pilot regarding his proposed course.

The grounding MPF profile (figure 5) highlighted important latent pathway factors as training (10.5\%), planning (9.6\%) and rules and policies (8.4\%). Active pathway factors were training (10.7\%), pressures (9.3\%) and planning (9.2\%). Further, an aggregate profile revealed communication (12.2\%) as an important factor, spread fairly evenly between active and latent pathways.

Insert figure 5 about here.

\section{Groundings: Generalised Accident Scenario}

The groundings analysed presented a coherent picture of the causal patterns involved. These could be brought together in a generalised grounding scenario (figure 6). Grounding was often the product of poor passage planning, which breached regulatory defences, in coincidence with one or both of a hazard identification failure and supervisory deficit. Whilst planning failure had causes immediately localised in the individuals committing---or omitting---them, they could be reliably traced to more distal precursors and organizational factors.

Wrong plans were due to mistakes. But these were dependent on inappropriate or less-than-adequate (LTA) information: vessels often had out of date, or no, charts for the channels they were using. Vessels are obliged to carry a set of charts detailing their proposed passage. However, routes were often altered ad hoc by charter companies once vessels had set sail. Further, requests by Masters to acquire charts at short notice were rarely supported by organizational structures. In two cases, for instance, company offices were shut for the weekend whilst Masters were contracted not to miss scheduled docking slots. So, inadequate information could be traced to poor organizational planning and deficient policies.

Incomplete or absent planning is a violation. However, time pressures and Pilot's familiarity with port approaches often rendered such violations necessary to meet deadlines, and were rarely perceived as risky. These factors can be traced to organizational pressures---in the form of recent deregulation and under-manning--and inadequate training. To correct such habitual behaviour, extensive and prolonged training would be necessary [18]. 
Poor planning on its own was, however, not enough to lead to grounding. Such inadequacies can presumably be compensated for in forgiving circumstances. Planning failures were found in association with hazard identification and supervisory deficits.

Hazard identification took the form of failures in correctly locating the vessel or channel. These were due to misdiagnoses or erroneous decisions. Again, precursors can be traced to fatigue, poor charts and inadequate plotting of vessel position (i.e. information less than adequate). Personal fatigue was often the result of long work hours resulting from organizational pressures and poor planning of shifts. Chart problems have the same causal roots as outlined above; that is, poor organizational planning and pressures. Errors in position plotting per se were rare, but plotting was often irregular or not accurate enough. This was attributed to poor training and rules regarding the level of position plotting necessary in close-shore situations.

Supervisory deficits were either communication or team support failures. These respectively resulted in information not being passed on, and disrupted cohesive BRM. Missing information was attributed to incomplete procedures for relaying details and a lack of knowledge regarding how and why this should be done. These precursors were therefore traced to rules, and training. Inadequate procedures and knowledge equally contributed to poor BRM, which was further compromised by under-manning, in turn due to organizational pressures. Further, both communication and BRM were often compromised by crews of various nationalities, with different native tongues. These crews had rarely received training to allow effective communication.

Supervisory failures prevented harnessing the combined cognitive resources of the bridge team to their full. This allowed individual unsafe acts to pass unnoticed and uncorrected, reducing the safety margin in these distributed team activities. As such, communication problems may best be thought of as interacting with planning or hazard identification performance, rather than as a separate and isolated causal chain.

Insert figure 6 about here. 


\section{Collisions}

Of the collisions analysed, the majority (60\%) involved incidents between a bulk carrier or cargo vessel and a trawler. 20\% were collisions due to drifting off anchor, and 20\% involved tug operations. First, it is worth briefly considering the latter two types of collision. Collisions by drifting off an anchorage were attributed to the crew on duty either not noticing ship movements, or noticing them but believing them to be instrument error without seeking corroborating evidence. These failures may be put down to the $86.5 \%$ peak for training on the drifting MPF profile. It could be argued that vessels should be obliged to anchor at greater distances from each other, so representing a policy failure. However, suitable anchorage grounds close to ports are not abundant, and the prescribed regulations allow good time to power the engines and correct the drift if an adequate watch is kept. Collisions between tugs and vessels they were assisting were due almost exclusively to temporary performance variability on the part of the tugmaster. Working in close quarters at low power, such collisions may be considered---to an extent---inevitable and relatively mild [20]. Whilst the above two incident types did have causes reaching beyond the active failures briefly described, due to the prominence and potential severity of bulk/cargotrawler (B/C-T) collisions this type is focused on here. Further, the ATSB have highlighted these incidents as ones of great concern [21], and have suggested that unreported near-miss situations are even more common than the twenty one actual collisions investigated between 1991 and 2001 would suggest.

\section{Human factor analysis}

$\mathrm{B} / \mathrm{C}-\mathrm{T}$ collisions were due to inadequacies of planning or conducting watch keeping on both vessels. All occurred at night, whilst watch was kept by just one crew member. CREAM analysis identified three important triggering failures. These were inadequate planning (38.1\%), inadequate observation (33.3\%) and poor interpretation (19\%), accounting for $90.4 \%$ of all proximal antecedents. These three main initiating factors were all individual cognitive failures. The event trees demonstrated that no single factor was sufficient in isolation. Planning in combination with observation or interpretation failures were sufficient triggers in $44.4 \%$ and $33.3 \%$ of the collisions respectively, accounting for $77.7 \%$ of the $\mathrm{B} / \mathrm{C}-\mathrm{T}$ collisions. 


\section{Planning errors}

The type of planning failure to occur on each of the two types of vessel involved in a $\mathrm{B} / \mathrm{C}-\mathrm{T}$ collision was consistently different, while observation and interpretation failures were common to both classes of vessel.

On the trawlers, planning inadequacies took the form of either no watch, or an inadequate watch, being planned. On three occasions, small trawlers' crews anchored their vessel and then all retired to bed. Alternatively, larger trawlers' lookouts were often planned to include an untrained or inexperienced crew member---on one occasion, for instance, a Mate's wife who had come along simply to cook the meals. Such small vessels also rarely had explicit procedures for keeping watch. Further, cognitive biases whereby the risk of a situation was not properly assessed were antecedent to just under one-half (44.4\%) of the incorrect watch keeping plans aboard trawlers. All vessels are required to keep an adequate watch at all times, so the above constituted violations, and accounted for $57.1 \%$ of all the violations identified by MAIT. These violations were routine, arising from under-manning and the lack of adequately trained members in such small crews.

Planning deficits on the bulk/cargo vessels took the form of inadequate clearance distances being planned when trawlers were being overtaken. Passing vessels are required to keep clear. As such, these poor plans too were violations. These were regularly attributed to one or both of two precursors. First, the watchman was inexperienced at judging and planning clearance distances at night. Second, the watchman was insensitive to the potential risk (30.8\% of all MAIT personal factors) due to the forgiving open-sea conditions. As such, these violations were erroneous, as the perpetrator did not actively seek to infringe the regulations.

\section{Observation and interpretation errors}

Observation failures consisted solely of missed observations, that is, not noticing the other vessel. They were mainly due to the dark night-time conditions (100\%), distraction by competing tasks (42.9\%) and insufficient experience (28.6\%). Interpretation failures all involved the faulty diagnosis of the other vessel's speed or heading. These were due to the watchmen's lack of knowledge regarding night time judgements (33.3\%), ambiguous information from visual and electronic bearings (33.3\%) and cognitive confirmation bias, where watchmen sought the minimum evidence to suggest the vessels were not on a collision course (33.3\%). These findings were supported by MAIT analysis, which isolated inexperience at night watch as 
46.2\% and over-reliance on one source of information as $23.1 \%$ of all personal factors. Further, $62.5 \%$ of errors identified by MAIT were those of poor and technologically unassisted judgements of the other vessel's heading and speed.

\section{Defensive and organizational factors}

MAIT analysis isolated two defensive mode failures of importance: rules (43.8\%) and hazard identification (31.2\%). 42.8\% of breaches of rule defences were by inadequate watch plans, whilst $28.6 \%$ were accounted for by incorrect passing clearances being planned. Hazard identification failures were fairly evenly split between improperly manned watches and the inadequate use of electronic equipment.

The B/C-T MPF profile (figure 7) highlighted important latent and active pathway factors as training (27.4\% and $45.2 \%$ respectively) and rules and policies (37.\% and 19.1\% respectively). Further, aggregate scores showed pressures to be the third most highly rated MPF at $23.8 \%$.

Insert figure 7 about here.

\section{Collisions: Generalised Accident Scenario}

The above analysis can be integrated into a comprehensive generalised collision scenario (figure 8). B/C-T collisions arose from deficient watch keeping on both vessels. Necessary failures were the breaching of rules specifying safe watch keeping or passing distance, on either---or both---the trawler and bulk/cargo vessel respectively, in conjunction with deficient hazard identification on either or both vessels.

Hazard identification failed either by entirely missing the presence of another vessel, or by misjudging its speed or heading. These in turn were both dependent on poor visibility at night, inexperience on the part of the watchman and the availability of poor or ambiguous information. The former is unavoidable, and was exacerbated by the later two factors. These had their roots in inadequate training and underspecified rules regarding the level of experience required for watch duty and the level of information needed to base sound judgements on at night. Further, missed observations were also often due to distraction by competing tasks as a result of an under-manned watch. Misdiagnoses were additionally due to confirmation bias, 
which could have been remedied by improved training and raising crews' awareness of this risk.

Rules regarding adequate watch keeping were routinely violated on trawlers. These violations had their roots in under-manning, absent or incomplete procedures and cognitive bias. In turn, links could be traced from these to organizational pressures, rules and policies and training. Passing clearance regulations were regularly erroneously violated on bulk/cargo vessels by crew inexperienced at planning safe passing manoeuvres and who were open to cognitive bias. These precursors had their roots in training deficiencies and, again, under-specified rules regarding watchmen's required level of experience.

Insert figure 8 about here.

\section{Conclusion}

Accidents at sea are costly events, so it is important to extract as much value from them as possible. As indicated previously, learning from accidents is aided by generalising from them, in order to uncover common patterns of failure that are likely to recur in other situations. This research identified common causal patterns in groundings and collisions, spanning defensive failures, unsafe acts, cognitive and situational precursors and deeper organizational factors. These findings resonate with and extend the previous studies published on the human factor causes underlying these types of accidents, and highlight a range of implications and potential applications of this approach.

The patterns identified by the research presented here were broadly in line with the key causes of groundings and collisions identified by previous studies [13, 14]. These studies predicted that groundings would often result from either a planning failure or a failure to properly identify or act on a deviation from a safe path, and that collisions would result from a failure to keep adequate watch. These immediate causes are relatively unsurprising and fit within the Generalised Accident Scenarios developed here. However, this research also identified supervision and team communication issues as an important contributing factor to groundings---likely due to the relatively fast-paced, collective and precise nature of the work needed to maintain a safe path in close-shore situations. Breakdowns in the sharing of information or in monitoring the activities of others could have immediate impacts in these circumstances. In 
contrast, the context in which collisions occurred typically involved only one person on each vessel on watch keeping and navigation duty.

Breaking these causal factors down further, previous research [10] pointed to a range of causes that were explored further by the analyses presented here. These included inaccurate position finding and little use being made of navigation aids. This was a significant factor in both GAS presented here. Crew were often found to rely on their own judgement to determine passing clearances, even when they were inexperienced in this area. The inadequate use of navigation aids often implicated in groundings commonly related to poor position plotting, inadequate use of radar---or that the required charts were simply not available. Dealing with these issues requires addressing faults deeper in the organization, primarily involving the careful design and delivery of both training and the resources required for each job. Previous studies $[9,10]$ also suggested that communication on the bridge and bridge layout are often inadequate for the tasks being conducted. Issues relating to bridge layout were often involved in both types of accident. In particular, the location of the chart room typically took members of the team away from their stations---a frequent problem in collisions. Rather than simply a physical design failure, however, the findings here identify the human and organizational roots of this problem. An adequate watch and team communication can be maintained if, for instance, training ensures that position plotting can be conducted swiftly and accurately and Bridge Resource Management processes are used to maintain effective information sharing [19].

More broadly, the findings presented here suggest that this structured approach to developing Generalised Accident Scenarios is useful for understanding and learning from past accidents. The value of the Generalised Accident Scenarios developed here lies in breaking down and specifying the human and organizational causal chains that typically lead to certain types of accidents. While the general scenarios may seem relatively complex and highlight many issues, in practice this translates into a wide range of opportunities to intervene in the development of accidents. This analysis suggests that breaking any one of these necessary error chains would forestall an accident. Modelling these causal patterns in detail provides crew, managers and policymakers a range of points at which to focus risk management action. Crew members could be trained to recognise and rapidly respond to the immediate failure modes and unsafe acts likely to cause an accident. Masters and company managers should be mindful of the precursors and organizational factors that can provoke those unsafe acts. And policymakers ought to focus on developing regulations and 
standards that address failures throughout the whole of these accident scenarios. This potentially allows more comprehensive policy responses than those that may result from single events $[22,23]$.

This approach, however, is not without its limitations. In terms of the analytical process, there is a degree of creativity and judgement required in constructing the causal patterns. Integrating the information from both analytical tools across many accidents requires time and some experience in accident analysis. The sort of analysis also requires a relatively high level of detailed information regarding individual accidents. It depends on prior detailed investigative work having been conducted into both the technical and human factors underlying accidents. This level of information is not always available. Further, it must be emphasised that the causal patterns produced do not represent deterministic and certain connections between causal factors. Rather, they highlight the most likely, logical and frequently observed interactions found within the set of accidents sampled. That is, a GAS provides a useful heuristic device, or rule-of-thumb, that helps to highlight key issues and direct attention, but does not claim to produce an objective and comprehensive accident model.

Generalising from marine accidents presents many opportunities for future research and application. Extending the analysis to other accident types would be welcome, as would be further efforts to develop and refine the scenarios developed here, for instance by integrating findings from a wider range of accident investigations produced by other organizations. Moreover, these accident scenarios could function as 'living documents', with findings from new accidents integrated into them as they become available. In this way, policymakers could maintain a database of generalised accidents across each type, amending and developing them in light of new experiences. It would also be interesting to assess the benefits and practicalities of company managers applying this approach on their own unique set of risk events, potentially producing a highly actionable set of findings. Applied and developed in these ways, it is to be hoped that this approach to constructing Generalised Accident Scenarios may be able to move beyond simply explaining why ships sometimes don't pass in the night. 


\section{References}

1. NATIONAL RESEARCH COUNCIL, 1976, Human Error in Merchant Marine Safety (Washington D.C.: National Academy Press).

2. MOORE, W. H., and BEA, R. G., 1993, Management of human error in operations of marine systems. Department of Naval Architecture and Offshore Engineering Report HOE-93-1, University of California, Berkeley.

3. GRABOWSKI, M., AYYALASOMAYAJULA, P., MERRICK, J., and MCCAFFERTY, D., 2007, Accident precursors and safety nets: leading indicators of tanker operations safety. Maritime Policy and Management, 34(5), 405-425.

4. REASON, J. T., 1997, Managing the Risks of Organizational Accidents (Aldershot: Ashgate).

5. HAVOLD, J. I., 2000, Culture in maritime safety. Maritime Policy and Management, 27(1), 79-88.

6. REASON, J. T., 1990, Human Error (Cambridge: Cambridge University Press).

7. PERROW, C., 1999, Normal Accidents: Living with High-Risk Technologies (Princeton: Princeton University Press).

8. WAGENAAR, W. A. and GROENEWEG, J., 1987, Accidents at sea: multiple causes and impossible consequences. International Journal of Man-Machine Studies, 27, 587-598.

9. GROENEWEG, J., 1994, Controlling the Controllable (Leiden: DSWO Press).

10. STOOP, J., 1998, Accident scenarios as a tool for safety enhancement strategies in transportation systems. In: After the Event: From Accident to Organizational Learning, edited by A. Hale, B. Wilpert and M. Freitag (Oxford: Pergamon), pp. 77-112. 
11. HARRALD, J. R., MAZZUCHI, T. A., SPAHN, J., VAN DORP, R., MERRICK, J., SHRESTHA, S. and GRABOWSKI, M., 1998, Using system simulation to model the impact of human error in a maritime system. Safety Science, $\mathbf{3 0}$, 235-247.

12. KIRWAN, B., 1994, A Guide To Practical Human Reliability Assessment (London: Taylor and Francis).

13. TECHNICA LTD, 1985, Prediction of the proportions of errant vessels in traffic lanes on the United Kingdom continental shelf. Offshore Technology Report OTH 85 214, Department of Energy, London.

14. AMROZOWICZ, M. D., 1996, The quantitative risk of oil tanker groundings. Unpublished thesis, Department of Mechanical Engineering, Massachusetts Institute of Technology.

15. HOLLNAGEL, E., 1998, Cognitive Reliability And Error Analysis Method: CREAM (Oxford: Elsevier).

16. REASON, J. T., FREE, R., HAVARD, S., BENSON, M. and VAN OIJEN, P., 1994, Railway Accident Investigation Tool (RAIT): a step-by-step guide for new users. Department of Psychology, University of Manchester.

17. FORSYTH, C. J., 1991, Factors affecting tanker safety. Maritime Policy and Management, 18(4), 313-319.

18. WAGENAAR, W. A, 1996, Profiling crisis management. Journal of Contingencies and Crisis Management, 4, 169-174.

19. ADAMS, M. R., 2006, Shipboard Bridge Resource Management: A Study of Human Factors Aboard Ship (Eastport: Nor'easter Press).

20. AUSTRALIAN TRANSPORT SAFETY BUREAU, 1998, Investigation into the collision between the Australian Flag tanker Barrington and the tug Austral Salvor. Maritime Safety Investigation Report 132. 
21. AUSTRALIAN TRANSPORT SAFETY BUREAU, 2000, Australian Transport Safety Bureau annual review. Department of Transport and Regional Services, Canberra.

22. CHO, D., 2007, The effects of the M/V Sea Prince accident on maritime safety management in Korea. Marine Policy, 31(6), 730-735.

23. Ren, J., Jenkinson, I., Wang, J., Xu, D. L., and Yang, J. B., 2008, A methodology to model causal relationships on offshore safety assessment focusing on human and organizational factors. Journal of Safety Research, 39, 87-100.

\section{Acknowledgements}

I am grateful to Jim Reason for providing the 'Railway Accident Investigation Tool' and other materials adapted and used in this research, and for his generosity with ideas. 
